\title{
High-speed jet noise
}

\author{
Christophe BAILLY* and Kozo FUJII** \\ *Ecole Centrale de Lyon \\ 36 avenue Guy de Collongue, 69134 Ecully cedex, France \\ E-mail: christophe.bailly@ec-lyon.fr \\ ** Institute of Space and Astronautics Science, Japan Aerospace Exploration Agency \\ Sagamihara, Kanagawa, 229-8510, Japan
}

Received 7 September 2015

\begin{abstract}
Aeronautical and space applications, but more generally various engineering issues, are concerned with noise of high-speed jets. This comprehensive review provides a summary of noise generated by supersonic round jets, including mixing noise, Mach wave radiation, broadband shock-cell noise and screech tones, examines efforts to understand the physics involved, and introduces recent numerical and experimental developments regarding high speed jet noise. It outlines also aerospace applications with topics such as rocket supersonic jet impinging a flame deflector installed on launch pads.
\end{abstract}

Key words : Supersonic jet, Mixing noise, Mach wave, Instability wave, Shock-cell noise, Screech, Underexpanded / Overexpanded round jet, Impinging supersonic jet

\section{Introduction}

High speed jet noise have been the subject of many experimental and theoretical studies in the past, but current projects involving supersonic jet noise in aeronautical and space applications stimulate new studies in high-speed jet noise. Advances in the direct computation of aerodynamic noise and near-field acoustics, combined with the increase in computing resources, as well as numerous developments in aeroacoustics methods, make possible original investigations regarding noise generation mechanisms, the assessment of models and the optimized design of new systems. Spectacular progress has also been made in the experimental techniques and signal processing to characterize the jet flow and its acoustics. The complementarity between all these approaches is critical to develop insightful research.

The purpose of this paper is to provide an overall picture of noise generated by a supersonic jet, including mixing noise, Mach waves, broadband shock-associated noise and screech. The review concisely examines the efforts of recent work to identify noise generation mechanisms in high-speed jets. There are already several contributions in this direction, for example Seiner (1984), Tam (1995), Raman (1999) or Morris (2007) to mention a few. The reader is invited to refer to the numerous references cited in these reviews, as well as to references given in the papers that are here mentioned. Furthermore, numerical and experimental techniques themselves are deliberately not discussed in the following. Their importance is however well known, as the choice of a smaller model in terms of Reynolds number and the prescription of consistent boundary conditions. Finally, only round jets are considered in the present text. There are many similarities between round and rectangular jets, but also some differences.

The text is organized as follows. Noise of perfectly expanded jets is presented in Section 2, with the contribution of the turbulent mixing noise and of Mach wave radiation. Nonlinear effects are also emphasized. Section 3 deals with shock-cell noise, including the broadband and tonal components. Aero and space applications are finally illustrated in Section 4, with the case of a supersonic jet impinging an inclined flat plate, modeling the presence of a flame deflector on a launch pad. 

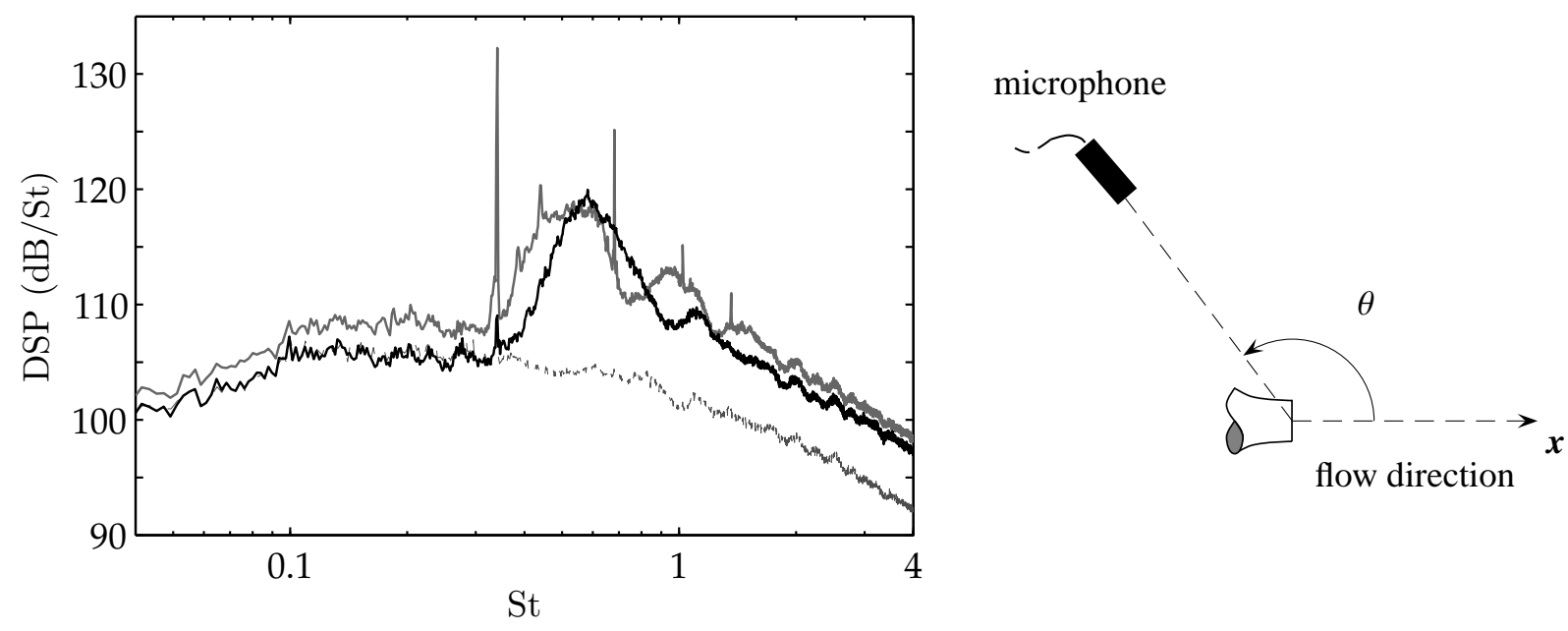

Fig. 1 Far field acoustic spectra in $\mathrm{dB} / \mathrm{St}$ measured at $r / D \simeq 52(D=38 \mathrm{~mm})$ for $M_{j}=1.30, T_{t}=T_{\infty}$, $R e_{D}=1.2 \times 10^{6}$ and $\theta=110^{\circ}$ as a function of the Strouhal number St: - convergent nozzle $\left(M_{d}=1\right)$, - notched convergent nozzle to remove screech (tonal component), and --- convergent-divergent nozzle $\left(M_{d}=M_{j}\right)$ to remove shock-cell noise. Data provided by André, Castelain and Bailly (2013). Note that the observer (listener) angle $\theta$ is taken from the jet flow direction $\boldsymbol{x}$.

\section{Noise of perfectly expanded supersonic jets}

A brief description of a supersonic jet flow is first presented in order to introduce the main parameters which will be used throughout this text. The jet operating point is determined by the nozzle pressure ratio (NPR), defined as the ratio between the reservoir or stagnation pressure $p_{t}$ and the ambient pressure $p_{\infty}$. The jet is sonic for a NPR greater than a critical value, given by $[(\gamma+1) / 2]^{\gamma /(\gamma-1)} \simeq 1.89$ for an ideal air flow, where $\gamma$ is the specific heat ratio. The jet can be equivalently characterized by its fully expanded Mach number $M_{j}=\left[2 /(\gamma-1)\left(\mathrm{NPR}^{(\gamma-1) / \gamma}-1\right)\right]^{1 / 2}$ assuming a quasi-onedimensional isentropic flow. This Mach number corresponds to the design Mach number $M_{d}$ of a convergent-divergent nozzle which should be considered to obtain a shock-free jet at this given NPR, and the exit pressure $p_{e}$ at the nozzle exit is then equal to the ambient pressure $p_{\infty}$. For a simple convergent nozzle, $M_{d}=1$, and the supersonic jet is underexpanded $\left(p_{e}>p_{\infty}\right)$ for NPR values larger than the critical value.

For a perfectly expanded high-speed jet, obtained consequently from a convergent-divergent nozzle operating at its design pressure ratio, jet noise is then only produced by turbulent mixing. As an illustration, acoustic spectra measured for three different nozzles at the same operating condition $M_{j}=1.30$, and for a given observer angle $\theta=110^{\circ}$ downstream from the jet axis, are shown in Figure 1. They are plotted as a function of the Strouhal number $\mathrm{St}=f D / u_{j}$, where $D$ is the nozzle throat diameter. The solid gray line corresponds to the noise radiated with a convergent plain nozzle, including mixing noise, screech and broadband shock-associated noise. Screech can be suppressed by using a notched convergent nozzle, as demonstrated by Bridges and Wernet (2008) or André, Castelain and Bailly (2013), and the experimental result is displayed in solid black line. It can be observed that the broadband shock-associated noise, and more globally the turbulent flow, is strongly affected by the presence of high-amplitude screech tones. This is discussed further in Section 3.2. The mixing noise component can be identified by operating a convergent-divergent nozzle at on-design Mach number, the measured acoustic spectrum is displayed in dashed gray line. Mixing noise does not appear to be modified by the broadband shock-associated noise, by comparison with the spectrum measured with the notched convergent nozzle in solid black line. This methodology for isolating mixing noise from broadband shock-associated noise, has been used by Tanna (1977a, 1977b) or Seiner and Yu (1984) for instance.

Initial conditions play a critical role on the turbulent development and acoustic field of jets. The influence of nozzleexit parameters such as the Reynolds number, the mean velocity profile or characteristics of the boundary layer have been identified as important parameters, mainly from experiments. To achieve highly controlled conditions, and thus carefully investigate effects of each parameter, high-fidelity numerical simulations appear, however, to be more attractive. Turbulent features in round jets are expected to be independent of the Reynolds number provided it is sufficiently high, classically for a threshold between $\operatorname{Re}_{D} \simeq 10^{5}$ and $4 \times 10^{5}$, corresponding to the turbulent transition of the boundary layer at the nozzle exit. The diameter-based Reynolds number is usually defined as $\operatorname{Re}_{D}=u_{j} D / v$ where $u_{j}, D$, and $v$ are the jet 
velocity, nozzle exit diameter, and kinematic molecular viscosity, respectively. As a result, a trip of the boundary layer is often required in numerical simulations to obtain turbulence levels in agreement with experiments at the nozzle exit, since the boundary layer developing inside the nozzle is generally poorly resolved for such values of the Reynolds number. Detailed reviews regarding flow development and sound emission can be found in Bogey et al. (2012, 2013).

\subsection{Turbulent mixing noise}

Mixing noise of supersonic jets can be first physically interpreted as a continuation of the subsonic regime as $M_{j}$ increases. Definition of jet noise sources has however been a long-standing controversy, refer to Tam et al. (1996), Morris (2007) and Tam et al. (2008a) for argumented discussions, but a widely shared view is that mixing noise is produced by two distinct noise mechanisms.

A first noise source is associated with the quasi-periodic passing of large-scale turbulent structures near the end of the potential core, where the flow is strongly intermittent and where the surrounding ambient fluid is entrained and accelerated inside the turbulent jet (Juvé et al., 1980; Camussi et al., 1999; Bogey \& Bailly, 2007; De Cacqueray et al., 2014). This source is predominant in the downstream direction, and can be modeled as the acoustic radiation of linear instability waves for convectively supersonic flows, as developed by Tam and Burton (1984). Further details concerning mathematical formulations, numerical results and references can be found in the two reviews provided by Tam (1995) and Morris (2010). Tam \& Hu (1989) carried out a comprehensive stability study of the dispersion relation for highspeed compressible jets. They found two additional families of wave solutions, beside the familiar Kelvin-Helmholtz instability waves. The first one corresponds to unstable acoustic modes propagating with a supersonic phase velocity with respect the ambient medium, when the jet velocity is larger than the sum of the speeds of sound inside and outside of the jet. These supersonic instability waves may also generate Mach waves. The second family corresponding to subsonic acoustic waves will be presented later. Remarkable experimental visualizations of the three families of instability waves and interpretations have been performed since the eighties by Oertel, Seiler \& Srulijes (2013).

For convectively subsonic flows, the initial growth of large scales is still linear, but the decay and breakdown of these structures is governed by the turbulent energy cascade involving nonlinear interactions. An experimental illustration is provided by Panda et al. (2005) with the correlation of a turbulent quantity in the jet plume with the far-field sound pressure fluctuations for subsonic and supersonic Mach numbers. Based on a Rayleigh scattering technique, measurements are nonintrusive and indicate a drop of the correlation for a subsonic convective Mach number. More recently, Sinha et al. (2014) have revisited radiation of instability waves in supersonic jets by solving the parabolized stability equations for a mean turbulent flow obtained by a high-fidelity large-eddy simulation. They found that compressible linear stability theory accurately describes the growing of flow disturbances, and its associated Mach wave radiation, but the agreement in the decay region is much poorer. Paradoxically, a single spectral shape of acoustic spectra is experimentally observed in the downstream direction, for subsonic and supersonic jets (Viswanathan, 2006).

The second source is linked to the turbulent mixing in the shear layer, and involves smaller turbulent scales, the so-called fine scales, by comparison to larger scales of coherent structures. This component has an almost isotropic directivity, is masked in the downstream direction but dominates in the sideline direction.

These two components of jet noise have been characterized by Tam, Golebiowski and Seiner (1996) and Viswanathan $(2004,2006)$ by examining a large experimental database of jet mixing noise. As an illustration, acoustic spectra measured at $\theta=30^{\circ}$ and $\theta=90^{\circ}$ are shown in Figure 2 for a perfectly expanded supersonic jet at $M_{j}=1.30$. The two semiempirical spectra associated with the two jet noise mechanims introduced above, are also plotted in dashed lines (Tam et al., 1996), by matching the frequencies and the peak levels. A combination of these two spectra should be applied to fit data at other angles. There is a difference of about $20 \mathrm{~dB}$ in amplitude, with a Strouhal peak of St $=0.17$ for the downstream acoustic radiation, and a Strouhal peak of $\mathrm{St}=0.29$ in the sideline direction. Expressions such as low- and high-frequency noise should thus be used with caution.

Numerical simulations play a primary role for studying jet noise mechanisms. The direct calculation of aerodynamic noise has indeed made spectacular progress over the two last decades. The increase in computational resources, advances in the development of low-dispersion and low-dissipation numerical schemes as well as in aeroacoustics methods have enabled to solve the unsteady compressible Navier-Stokes equations by including the acoustic field as part of the simulation. The goal of computational aeroacoustics should not be to provide only one large numerical experiment, but also to develop detailed studies, in order to identify and understand the role of various parameters such as Reynolds numbers, 


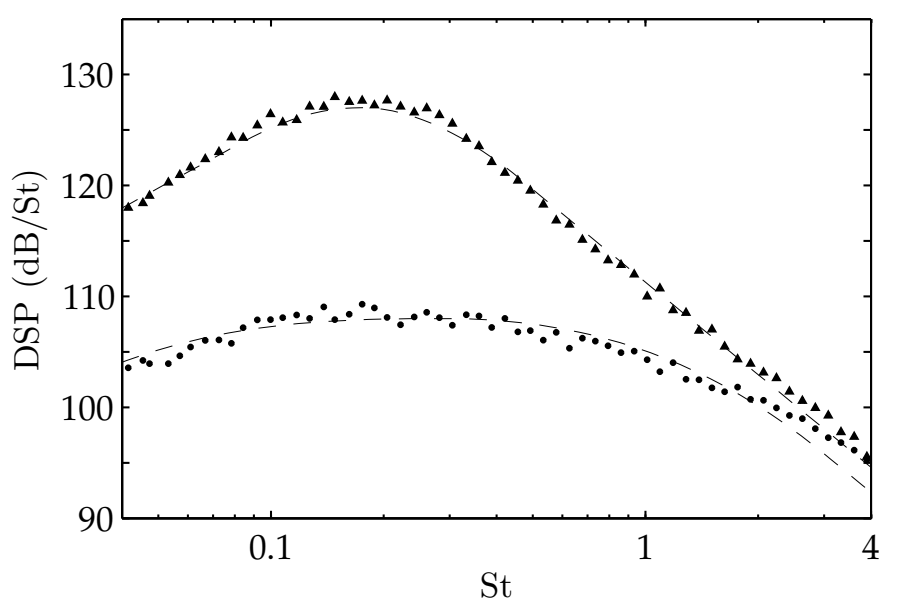

Fig. 2 Acoustic spectra measured at two observer angles, $\boldsymbol{\Delta} \theta=30^{\circ}$ and $\bullet \theta=90^{\circ}$, for a perfectly expanded supersonic jet at $M_{j}=1.30$, see Fig. 1 for more information. The two semi-empirical spectral shapes of Tam, Golebiowski and Seiner (1996) are also plotted as dashed lines. Data provided by André, Castelain and Bailly (2013).

see Bogey \& Marsden (2013) for instance, in a controlled environment. Jet noise sources can then be directly investigated by using the causality method in which cross-correlations between flow quantities inside the jet and the radiated sound pressure are calculated (Bogey \& Bailly, 2007), as carried out by De Cacqueray \& Bogey (2014) for an overexpanded high-speed jet. With the aim to compute noise produced by rocket jets, Nonomura and Fujii (2010) have investigated the influence of overexpansion effects on the radiation of Mach waves, keeping $M_{j}=3$ with $3 \leq M_{d} \leq 4$, by large-eddy simulation. They found that the formation of a Mach disk in the first shock-cell enhances shear-layer mixing, and that Mach waves are then emitted much closer to the nozzle. Another illustration has been recently provided by Ryu, Lele \& Viswanathan (2014). Using the large-eddy simulation data base by Bodony \& Lele (2005), they show that the supersonic wave components of the flow, responsible of Mach wave radiation, scale as the eighth power of the jet velocity.

Similar approaches have been also developed in experiments. Beamformed flow-acoustic correlations have been studied by Papamoschou et al. (2010) for a cold supersonic jet at Mach 1.75 for instance. Higher coherence levels are measured with respect to correlations between two single probes, and the contribution of large scales in the main radiation direction is stressed. Moreover, identification of large turbulent structures or instability waves as the dominant contribution for mixing noise in high-speed jet flows has motivated the development of active control for noise reduction, but also for the increase of mass entrainment or mixing enhancement. References can be found in Samimy et al. (2007) with the study of a round supersonic jet at Mach 1.3, and Sinha et al. (2010) for a Mach 0.9 jet at moderate Reynolds number.

\subsection{Nonlinear propagation effects and crackle}

Nonlinear effects are found to be quite significant in the sound propagation of high-speed jet noise (Gee et al., 2008). Waveform distortions and formation of weak shock waves leading to a series of $N$-shaped waves are clearly observed in measurements. They can be quantified from experiments, based on a generalized Burgers equation, as studied by Baars et al. (2014a) for a perfectly expanded laboratory jet at Mach 3. These authors provide guidelines to ensure that a model-scale and a full-scale jet will give same nonlinear propagation effects.

De Cacqueray and Bogey (2014) have investigated an overexpanded supersonic jet at Mach 3.3 by large-eddy simulation. They performed two far-field wave extrapolations from the near-field data, by solving the linearized Euler equations in one case, and the full Euler equations in the other. Pressure signals recorded at four distances from the nozzle exit, and for an observer angle close to the peak directivity angle, are shown in Figure 3. The numerical pressure signals determined at $r=30 \mathrm{D}$ from both acoustic computations are on the whole similar. Differences due to nonlinear effects can however be observed, as is the case at time $t-\tau \simeq 190$ for instance. As the propagation distance from the nozzle exit increases, the changes between the two signals are stronger. The pressure signal calculated using the full Euler equations gradually have a lower amplitude than that calculated using the linear Euler equations. Weak shock waves also appear in the nonlinear case, leading to a series of $N$-shaped waves in the signal at $r=120 \mathrm{D}$ in Figure 3(d). Consequently, far-field acoustic spectra are affected with an energy transfer from the spectral peak to the higher frequencies as the propagation 
(a)

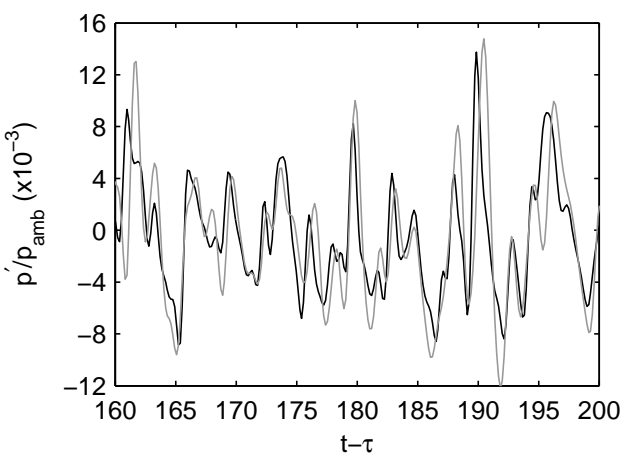

(c)

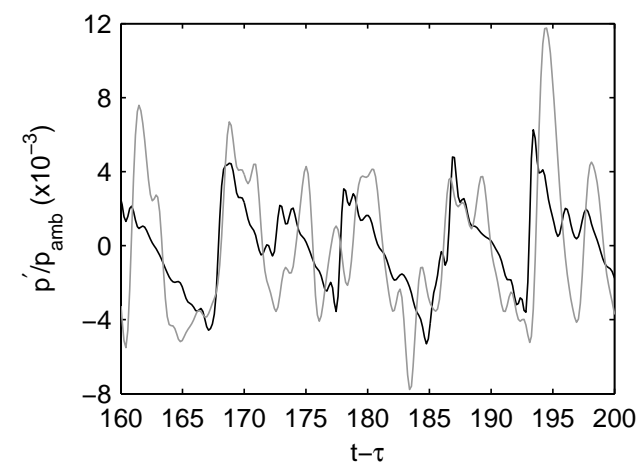

(b)

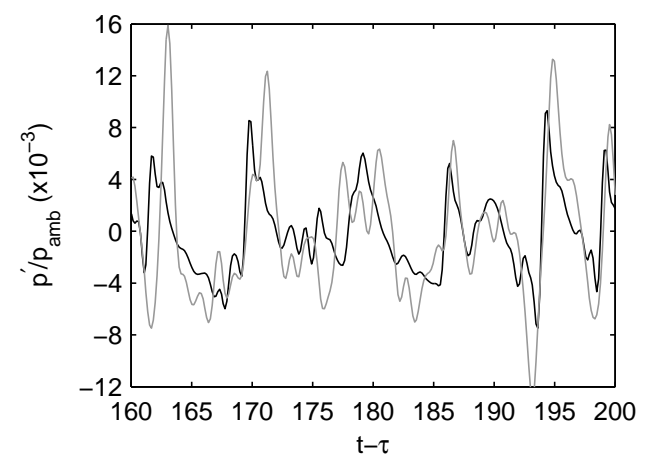

(d)

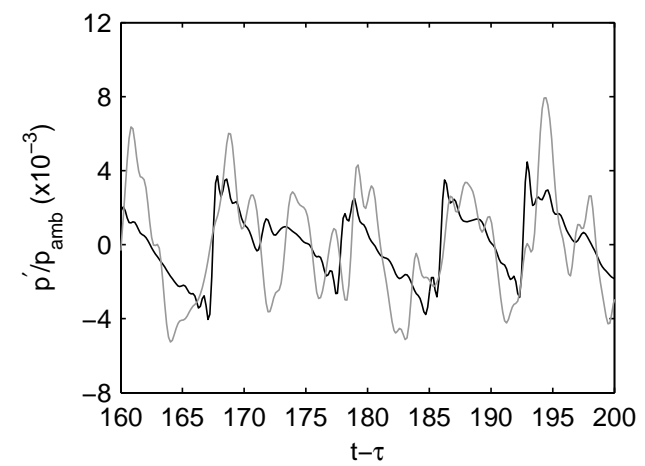

Fig. 3 Large-eddy simulation of an overexpanded Mach 3.3 jet, with a Reynolds number $\operatorname{Re}_{D}=9.4 \times 10^{4}$ and $T_{t} / T_{\infty}=3.9$, where $T_{t}$ and $T_{\infty}$ are respectively the stagnation and ambient temperatures. Dimensionless signals of pressure fluctuations obtained for $\theta=60^{\circ}$ at (a) $r=30 D$, (b) $r=60 D$, (c) $r=90 D$ and (d) $r=120 D$ from the nozzle exit: far-field extrapolation from the near-field data based on - the linearized and - the full Euler equations. Time $t$ and time delay $\tau=r / c_{\infty}$ are normalized by $u_{j} / D$, where $c_{\infty}$ is the ambient speed of sound. From de Cacqueray \& Bogey (2014)

distance increases. As an illustration, the peak level observed around $\theta=60^{\circ}$ is reduced by about $5 \mathrm{~dB}$ at $r=120 D$ for this laboratory jet $(D=32 \mathrm{~mm})$.

These acoustic sawtooth-like structures generate a specific noise, known as the crackle sound, perceived in the farfield of supersonic jets. Crackle can be characterized by estimating indicators of nonlinearity such as the skewness values of pressure signals, but also by using more elaborated algorithms (Mora et al, 2014). A recent review and study is provided by Baars and Tinney (2014b). Crackle appears to be sensitive to nozzle lip modification or to the presence of chevrons, which suggest that this particular noise would be generated by the turbulent flow itself, and not by nonlinear effects during the far-field propagation (Nichols et al., 2013).

\section{Noise of shock-containing supersonic jets}

With the presence of a shock-cell structure in the supersonic jet core, an additional component to the mixing noise is produced, as already illustrated in Figure 1. For a convergent nozzle, the Mach number at the nozzle exit is still equal to unity for higher values of NPR, but the exit pressure $p_{e}$ no longer matches the ambient pressure $p_{\infty}$. As a result, a weak shock-cell structure appears inside the transonic jet to adapt the pressure field to this ambient pressure, as shown schematically in Figure 4, and the jet is said underexpanded since $p_{e}>p_{\infty}$. The opposite situation occurs in overexpanded jet. Shock noise is composed of screech, characterized by high-amplitude discrete tones, and of broadband shock-associated noise, characterized by a broadband hump dominating mixing noise in the upstream direction. These two additional components of turbulent mixing noise can be clearly identified in Figure 1.

\subsection{Broadband shock-cell noise}

Harper-Bourne \& Fisher (1973) provided one of the first comprehensive description of broadband noise by shockcontaining jets. Their model is based on a phased array of monopoles along the jet axis, by assuming that noise is generated when shock tips intersect the jet shear layer, and that adjacent sources of the quasi-regular shock-cell structure 


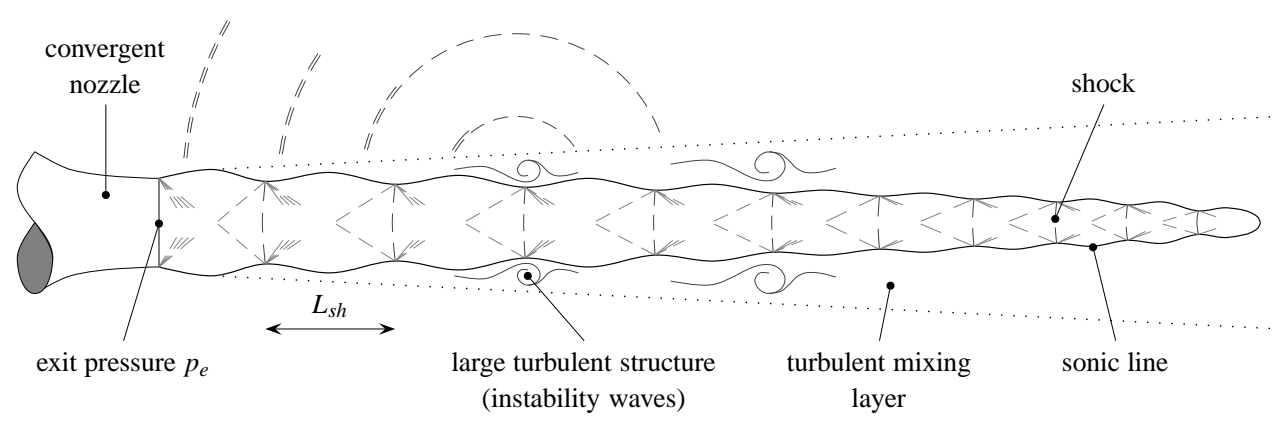

Fig. 4 Diagram of underexpanded supersonic jet illustrating screech noise. The quasi-periodic shock-cell structure is generated by expansion fans from the nozzle lip, which reflect on the sonic line boundary of the jet plume. The feedback wave is often generated between the second and the fifth shock cell by shock - vortex interaction, the location resulting of a balance between the growth of instability waves from the nozzle lip and the strength of the shock-cells. The acoustic wave travels toward the nozzle lip, and the resonant screech loop is maintained by a receptivity mechanism at the nozzle.

are correlated (Harper-Bourne, 2002). The far-field results from interference of these sources, with a peak frequency for the hump given by

$$
f_{p}=\frac{u_{c}}{L_{s h}\left(1-M_{c} \cos \theta\right)}
$$

where $u_{c}$ is the convection velocity of large structures, $L_{s h}$ is the mean shock cell-spacing, $M_{c}=u_{c} / c_{\infty}$ is the convection Mach number and $c_{\infty}$ is the ambient speed of sound. The peak frequency $f_{p}$ increases as the observer angle $\theta$ decreases toward the jet axis direction, and the Doppler factor reflects convection of flow disturbances along consecutive shock-cells. An estimation of $L_{s h}$ is provided by Tam et al. (2014), $L_{s h} \simeq 1.18 \beta D_{j}$ where $\beta=\left(1-M_{j}^{2}\right)^{1 / 2}$ and $D_{j}$ is the fully expanded jet diameter at $M_{j}$. The reader may refer to Powell (2010) for a complete discussion regarding various Prandtl's formulas for the cell length $L_{s h}$. It can be noted from Eq. (1) that $f_{p}$ diminishes with increasing nozzle pressure ratio, as a result of the shock-cells lengthening. Broadband shock-associated noise reaches its maximum in the upstream direction. HarperBourne \& Fisher also noticed that over a wide range of Mach numbers, noise intensity varies as $I \propto \beta^{2}$ for convergent nozzles. Furthermore, broadband shock-cell noise sources appear to be located relatively far from the nozzle exit (Seiner, 1984).

The directivity of shock noise emission can also be modeled in considering the interaction of instability waves, represented as a progressive wave in the jet flow direction $x$, that is $u_{t} \sim e^{i(\alpha x-\omega t)}$ where $\alpha \simeq \omega / u_{c}$ is the axial wavenumber and $\omega=2 \pi f$ the angular frequency, with the quasi-periodic steady shock-cell structure $u_{s h} \sim \cos \left(k_{s h} x\right)$ with $k_{s h}=2 \pi / L_{s h}$, as developed by Tam and Tanna (1982). The resulting source term for shock-cell noise generation is then given by

$$
u_{t} u_{s h} \sim \underbrace{e^{i\left[\left(\alpha-k_{s h}\right) x-\omega t\right]}}_{W^{-}}+\underbrace{e^{i\left[\left(\alpha+k_{s h}\right) x-\omega t\right]}}_{W^{+}}
$$

The first term corresponds to a traveling wave $W^{-}$of phase velocity $v_{\varphi}^{-}=\omega /\left(\alpha-k_{s h}\right)$. For values of $k_{s h}$ slightly greater than $\alpha$, this phase velocity is negative and greater than the ambient speed of sound $c_{\infty}$. Mach waves can thus be generated in the upstream direction, as illustrated in Figure 5, with an emission angle such that $\cos \theta=c_{\infty} / v_{\varphi}^{-}$. Expression (1) of the frequency peak $f_{p}$ is recovered from this radiation condition. The spectral broadening around $f_{p}$ can be explained by the variations of the convection velocity and of the amplitude of the wavepacket, not introduced here. Regarding waves $W^{+}$in Eq. (2), the phase velocity $v_{\varphi}^{+}=\omega /\left(\alpha+k_{s h}\right)=u_{c} \omega /\left(\omega+k_{s h} u_{c}\right)$, is positive and always smaller than the convection velocity $u_{c}$, so less capable of radiating Mach waves. Furthermore, Tam \& Tanna (1982) showed that the acoustic intensity of broadband shock-associated noise is proportional to $I \propto\left(M_{j}^{2}-M_{d}^{2}\right)^{2}$ for a convergent-divergent nozzle, which generalizes Harper-Bourne \& Fisher's result for convergent nozzles. More detail experimental results can be found in Viswanathan $e t$ al. (2010) and André et al. (2013).

Various engineering issues are concerned with shock-cell noise, including acoustic fatigue for military aircraft and interior noise for civil aircraft. In the latter case, the secondary stream of commercial engines is underexpanded during the climb and cruise phases of a flight, as illustrated in Figure 6. Tam, Pastouchenko and Viswanathan (2008b, 2009a) have numerically generalized the previous analytical approach by solving the linearized Reynolds-Averaged Navier-Stokes equations to compute the noise of dual-stream jets issued from a separate nozzle. Morris \& Miller (2010) have recently 


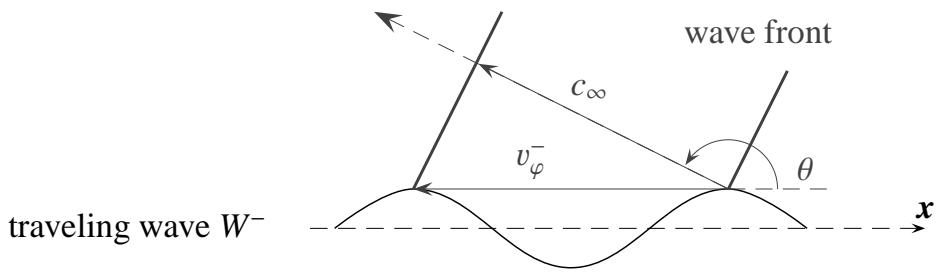

Fig. 5 Generation of Mach waves in the upstream direction by a traveling supersonic wave with a negative phase velocity (Tam, 1995). The direction of radiation $\cos \theta=c_{\infty} / v_{\varphi}^{-}$is obtained by matching the velocity trace along the flow direction $\boldsymbol{x}$.

developed a numerical model to predict shock-cell noise, in extending what has been done for mixing noise in the framework of statistical modelling, and by including flight effects in the context of aeronautical applications. The inputs of such models are provided by a Reynolds-Averaged Navier-Stokes solution. Role of the nozzle contour on acoustics and thrust has been investigated by Cuppoletti et al. (2014) from large-eddy simulations and comparison with measurements. They showed through improved nozzle design, that the jet thrust can be significantly increased while avoiding acoustic penalties. Measurements for conical convergent-divergent nozzles can also be found in Munday et al. (2011). The use of chevrons combined with a fluidic injection is considered in Heeb et al. (2010)
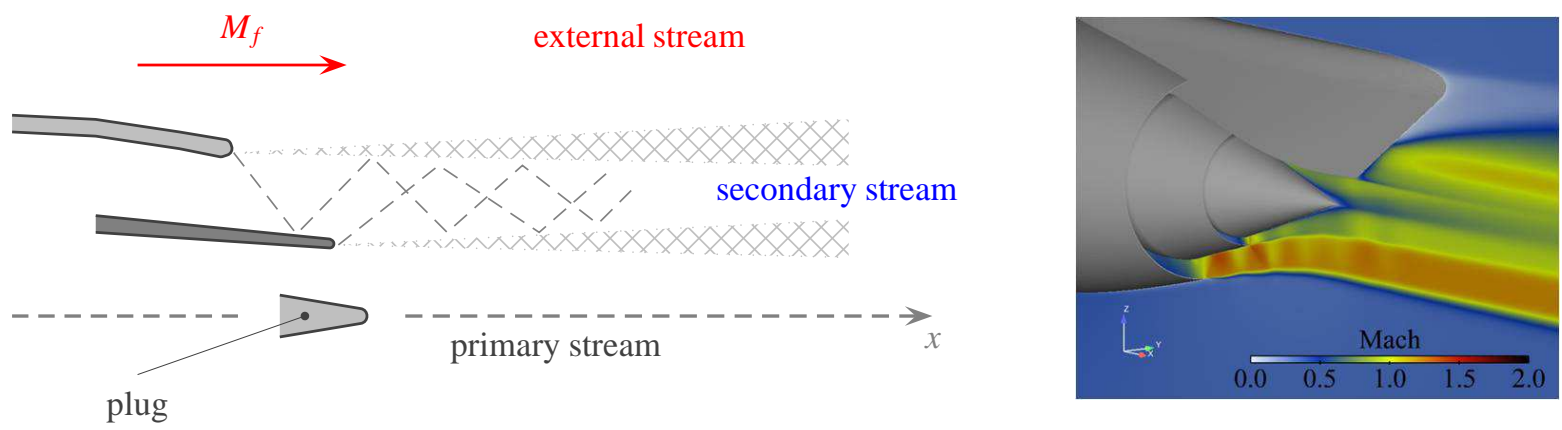

Fig. 6 Diagram of the exhaust of a commercial engine with a high bypass ratio, consisting of a central plug, the primary hot jet, the secondary cold underexpanded jet and the external stream characterized by its flight Mach number $M_{f}$ at cruise condition. The Mach number field taken from a RANS simulation of a generic configuration is also shown (Courtesy of C. Henry, Snecma). The shock-cell structure in the secondary stream can be clearly seen.

\subsection{Screech}

Noise of shock-containing supersonic jets has a broadband component, and has also a screech component constituted of a few discrete tones, which can be easily identified in the full spectrum (solid gray line) of Figure 1. On the contrary to broadband shock-cell noise, tone frequencies are however independent of the observer position. Screech is generally observed for laboratory jets and on military twin-engine aircraft for instance, but not on civil aircraft engines. As already mentioned in Section 2, screech is however known to have a strong impact on the turbulent flow dynamics and the radiated sound field, its suppression is consequently an essential effort for only studying broadband shock-associated noise.

Screech has been discovered by Powell (1953) for rectangular and round choked jets, and a review is provided by Raman (1999). This tonal noise is induced by a feedback mechanism along the mixing layer of the jet. Vortical structures are generated at the nozzle lip, and are convected downstream in the mixing layer. These perturbations interact with shock-cells and create acoustic waves that propagate upstream to the nozzle lip in the ambient medium, and then trigger subsequent instability waves, which form new vortical structures along the shear layer bounding the jet, as illustrated in Figure 4. This loop is resonant for some frequencies which are the fundamental screech frequency and its harmonics. Basically, four distinct modes associated with instability waves developing in the shear layer have been identified. Using the conventional terminology for a cold round jet issued from a convergent nozzle, axisymmetric modes $A_{1}$ and $A_{2}$ are observed for a fully expanded jet Mach number of less than about 1.3, see Powell et al. (1992), Ponton and Seiner (1992), Raman (1999) and André et al. (2011) for documented experiments among others. At higher Mach numbers, sinuous or flapping mode B and helical mode $\mathrm{C}$ can be identified, whereas mode $D$ is now classified as a sinuous/flapping mode. Thanks to progress in flow and acoustic measurements, these screech modes can be investigated in detail, see for 
instance Panda (1999), André et al. (2011) and Edgington-Mitchell et al. (2014) for circular jets. Moreover, various subtle behaviours occur regarding mode switching, rotation of the symmetrical plane, generation of the flapping modes, installation effects, flight effects or screech cessation. For the latter, Raman (1997) has shown that the excessive expansion of the jet boundary at high NPR may induce an aerodynamic blockage of feedback near the nozzle lip.

Powell proposed a simple acoustic model to explain the frequency selection. By considering the axisymmetric mode as an illustration, the pressure field results from a phased array of acoustic monopole sources, each fixed source being located at the end of a periodic shock-cell with a phase shift determined by a constant convection velocity (Norum, 1983). The screech frequency $f_{s}$ is obtained by imposing that all the acoustic waves reach the nozzle lip in phase, which provides

$$
\frac{n}{f_{s}}=\frac{L_{s h}}{u_{c}}+\frac{L_{s h}}{c_{\infty}}=\frac{L_{s h}\left(1+M_{c}\right)}{u_{c}}
$$

where $n$ is an integer. In other words, the sum of the convection time of vortical structures along the shear layer plus the acoustical return time to the nozzle lip is an integer of the screech oscillation periods. The directivity of the fundamental and its first harmonic are also well estimated with this model. A more detailed comparison with experiments is however not in favor of the model. For instance, the convection velocity is not uniform as expected, and the frequency is actually not accurately predicted for the different modes. Furthermore, screech emission is found to be localized to a few shockcells, or even a single shock-cell, between the third and the fifth shock. Powell, Umeda and Ishii (1992) have revisited this model leading to Eq. (3). The source location is now assumed to be at a distance $h$ from the nozzle, with $n$ vortical structures along this distance $h$. In a similar way to edge tones or Rossiter modes for a cavity, the frequency is then given by

$$
\frac{n}{f_{s}}=\frac{h}{u_{c}}+\frac{h}{c_{\infty}}=\frac{n_{s h} L_{s h}\left(1+M_{c}\right)}{u_{c}}
$$

where $h=n_{s h} L_{s h}$ provides the screech source position as the $n_{s h}$-th shock-cell. Based on unsteady Reynolds-averaged Navier-Stokes simulations, Gao and $\mathrm{Li}$ (2010) have identified the two integers $n$ and $n_{s h}$ for various modes of their computations.

Tam, Seiner and Yu (1986) consider screech is a special case of broadband shock-cell noise. The mechanism involved in noise emission is basically not different of that discussed in Section 3.1 for their shock-cell noise model, and they point out that $f_{p} \rightarrow f_{s}$ as $\theta \rightarrow \pi$. Based on numerical simulations, Shen and Tam (2002) (see also Tam et al., 2014), verified the feedback loop for various screech modes, by recasting Eq. (1) for $\theta=\pi$ or equivalently Eq. (3) in the following dimensionless form $k_{s h} D-\alpha D-k_{\infty} D=0$, and estimating each of the three terms. A good agreement is found for the $A_{1}$ and $B$ modes, but not for the $A_{2}$ and $C$ modes. For these modes, the acoustic feedback is attributed to a subsonic acoustic mode, associated with the third family of instability waves introduced in Section 2.1 for high-speed jets. This acoustic wave propagates outside of the supersonic core, in the upstream direction and guided by the jet. The two acoustic feedback mechanisms have been simultaneously observed, leading to two distinct frequencies of screech tones. For completeness, another point of view is chosen by Panda (1999), based on experimental observations of a stationary wave of wavelength $L_{s w}$ for the near pressure field. The characteristic length $L_{s h}$ is then replaced by $L_{s w}=2 \pi /\left(\alpha+k_{\infty}\right)$ where $k_{\infty}=\omega / c_{\infty}$.

Vortex-shock interaction leading to acoustic emission has been examined in detail by Suzuki and Lele (2003). They have shown that shock leaks through the shear layer near the saddle points of vorticity, and creates a pressure gradient that propagates in the near field with a quasi-circular wave front, covering a wide range of angles including the upstream direction. This shock-leakage mechanism has been identified in three-dimensional numerical simulations of a rectangular screeching jet by Berland et al. (1997), and screech source is found near the third shock-cell in this particular case.

\section{Aero and space applications}

High-speed jets are naturally present in aerospace applications. On aircraft carrier flight decks, the presence of tactical aircraft generates noise levels in excess of $150 \mathrm{~dB}$, inducing serious health and safety concerns for personnel. Jet blast deflectors are employed to divert the flow of high-temperature exhaust away for conventional takeoff and landing, but the jet impingement produces additional noise. Furthermore, intense tones can be generated thanks to self-sustaining acoustic feedbacks already mentioned in Section 3.2 for screech noise (see Henderson et al., 2005 and Gojon et al., 2015).

Another example is provided with rocket motors commonly used in aerospace applications. Such motors equip the launch vehicules, such as Vega and SRB's (solid rocket boosters) of Ariane 5 in Europe, SRBs of the Space Shuttle 
(a)

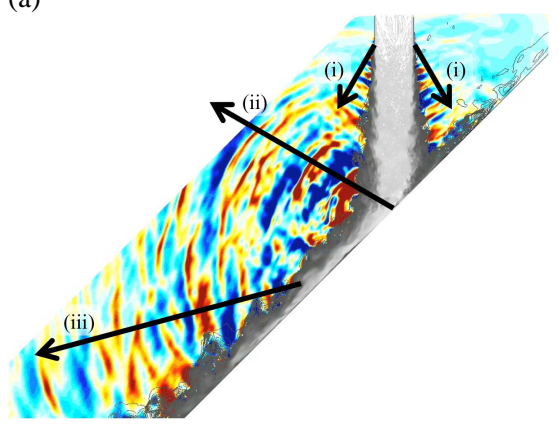

(c)

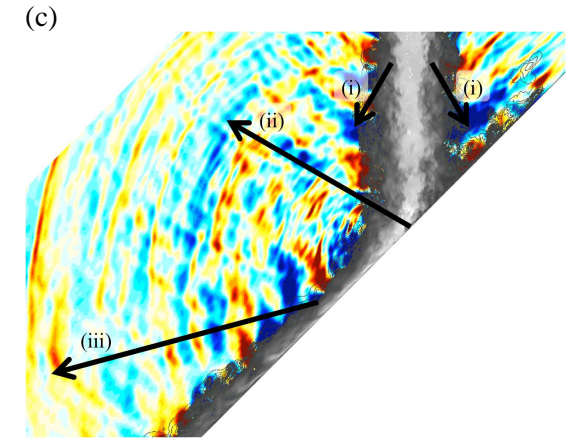

(b)

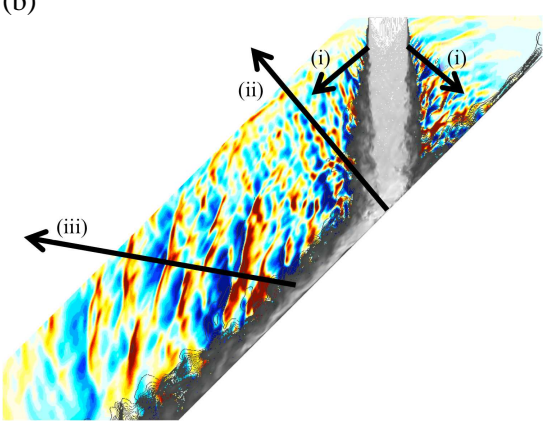

(d)

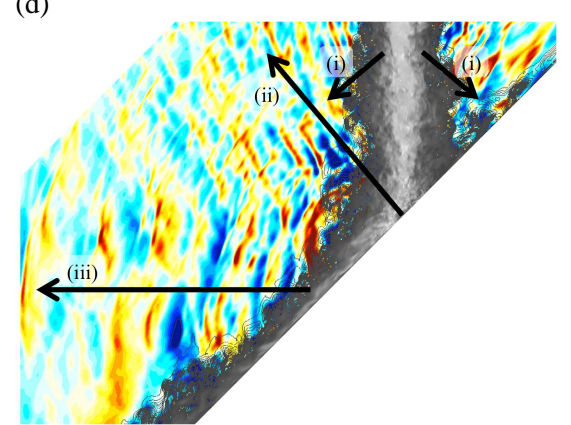

Fig. 7 Supersonic jet impinging on an inclined plate. Four snapshots of the acoustic pressure field (in color) and of the aerodynamic pressure field (in gray) computed by large-eddy simulation. Two distances between the nozzle and the impingement point are shown, $L / D=5$ for cases (a) and (b), and $L / D=10$ for cases (c) and (d). Furthermore, a cold jet is considered in cases (a) and (c) with $T_{t} / T_{j}=1$, and a hot jet in cases (b) and (d) with $T_{t} / T_{j}=5.4$. From Nonomura, Goto and Fujii (2011)

in the United States and Epsilon rockets and HIIA/B SRBs in Japan. When an SRB ignites, strong transient pressure waves are generated and expelled out the nozzle. These transient pressure waves are called ignition overpressure (IOP). When these motors ignite in a silo, the expelled pressure waves reflect from the silo floor and propagate up over the vehicle. When these motors ignite on an open launch pad, two types of pressure waves are propagated over the vehicle and surrounding structures, see Dargaud et al. (2013). In addition to IOP, which is a very strong single transient pressure wave, weaker pressure waves are also continuously emitted from the jet structure of rocket plumes during the rocket liftoff stage. Prediction of these weak pressure waves which are called rocket plume acoustics, is very important because both satellite and rocket vehicles are exposed to severe structural vibration environments induced by this sound field.

Design of a new launch pad with the goal of reducing noise generated by a rocket plume is obviously a great challenge, and required the use of computational aeroacoustics to aid in the development of noise reduction concepts. A comprehensive review of these computational studies for practical problems is provided by Fujii et al. (2010). Among the various topics, which must be solved, it is desirable to get a complete view of the sound source distributions when the supersonic jet impinges the deflector of the launch pad. An example of such studies is illustrated in Figure 7, where snapshots of the acoustic pressure field (in color) and of the aerodynamic pressure field (in gray) computed by large-eddy simulation (Nonomura et al., 2011) are shown for two geometrical configurations, and for a cold and a hot supersonic jet. For each pressure field, the authors indicate three source regions. Sound waves (i) and (iii) correspond to Mach wave generated by the supersonic jet, and by the supersonic boundary layer along the inclined flat plate, whereas sound waves are directly linked to the impingement noise. The influence of the temperature is also discussed in the study. More recent analysis by Honda et al. (2011) and Nagata et al. (2013) have identified two types of flow structures, which are responsible for noise generation of the sound waves (ii). A correct estimation of the low frequency noise perceived by the laucher is required to accurately describe and model these three source regions as a function of the distance between the nozzle and the impinging point. More recently, Tatsukawa et al. (2015) have performed a multiobjective aeroacoustic design optimization by evaluating 2500 configurations. The idea is to reduce the overall radiated noise by changing the curve of the deflector, but in also minimizing the deflector deformation with respect to a simple flate plate inclined at $45^{\circ}$. The authors show that the contribution of sound waves (ii) is mainly reduced.

In 2013, JAXA was successful in more than $10 \mathrm{~dB}$ SPL (sound pressure level) reduction for a newly-developed 
Epsilon rocket compared to previous $\mathrm{M}-\mathrm{V}$ rocket by the improvement of launching pad design of the Uchinoura space center. Computational studies by Tstutumi et al. (2015) contributed a lot for this reduction. Two space agencies, JAXA and CNES continue collaborative research for the better estimation of rocket plume acoustics. Recently, NASA becomes strongly interested in the estimation of rocket plume acoustics, as the new heavy-lift Space Launch System (SLS) is under development. With the progress of computer capability together with basic study for the nature of rocket plume acoustics, computational approach will contribute more and more to the estimation and reduction of rocket plume acoustics.

\section{Concluding remarks}

Significant work has been carried out during the last two decades to identify noise generation mechanisms in highspeed round jets, and these efforts are concisely examined in this review to provide a general picture of noise generated by a supersonic jet, including mixing noise, Mach waves, broadband shock-associated noise and screech, has been proposed. Progress in experimental techniques as well as in numerical methods and computing resources during this period are quite spectacular. New experiments and numerical simulations have thus led to some answers, some examples are mentioned in this review, but also to new questions, in particular for shock-cell noise, but other topics such as temperature effects can be mentioned. Moreover, various models of noise generation have been developed in aeroacoustics. An insightful general approach, however, should be able to describe and predict high-speed jet noise, but also to explain failures of previous work and experimental results. Such efforts are required for a better understanding of aerodynamic noise in general. Finally, developments presented in the last section illustrate a wide field of investigation for, at least, local noise reduction, and for which our knowledge in aeroacoustics are useful.

\section{Acknowledgment}

This work was supported by the Labex Centre Lyonnais d'Acoustique of Université de Lyon, operated by the French National Research Agency (ANR-10-LABX-0060/ANR-11-IDEX-0007).

\section{References}

André, B., Castelain, T. and Bailly, C., Experimental study of flight effects on screech in underexpanded jets, Physics of Fluids, Vol. 23, No. 126102 (2011), pp. 1-14.

André, B., Castelain, T. and Bailly, C., Shock oscillations in a supersonic jet exhibiting antisymmetrical screech, AIAA Journal, Vol. 50, No. 9 (2012), pp. 2017-2020.

André, B., Castelain, T. and Bailly, C., Broadband shock-associated noise in screeching and non-screeching underexpanded supersonic jets, AIAA Journal, Vol. 51, No. 3 (2013), pp. 665-673.

Baars, W.J., Tinney, C.E., Wochner, M.S. and Hamilton, M.F., On cumulative nonlinear acoustic waveform distortions from high-speed jets, Journal of Fluid Mechanics, Vol. 749 (2014), pp. 331-366.

Baars, W.J. and Tinney, C.E., Shock-structures in the acoustic field of a Mach 3 jet with crackle, Journal of Sound and Vibration, Vol. 333 (2014), pp. 2539-2553.

Beneddine, S., Mettot, C. and Sipp, D., Global stability analysis of underexpanded screeching jets, European Journal of Mechanics B/Fluids, Vol. 49 (2015), pp. 392-399.

Berland, J., Bogey, C. and Bailly, C., Numerical study of screech generation in a planar supersonic jet, Physics of Fluids, Vol. 19, No. 075105 (2007), 1-14.

Bodony, D.J. and Lele, S.K., On using large-eddy simulation for the prediction of noise from cold and heated turbulent jets, Physics of Fluids, Vol. 17, No. 085103 (2005), pp. 1-20.

Bogey, C. and Bailly, C., An analysis of the correlations between the turbulent flow and the sound pressure field of turbulent jets, Journal of Fluid Mechanics, Vol. 583 (2007), pp. 71-97.

Bogey, C., Marsden, O. and Bailly, C., Effects of moderate Reynolds numbers on subsonic round jets with highly disturbed nozzle-exit boundary layers, Physics of Fluids, Vol. 24, 105107 (2012), pp. 1-24.

Bogey, C. and Marsden, O., Identification of the effects of the nozzle-exit boundary-layer thickness and its corresponding Reynolds number in initially highly disturbed subsonic jets, Physics of Fluids, Vol. 25, No. 055106 (2013), pp. 1-27.

Bridges, J.E., and Wernet, M.P., Turbulence associated with broadband shock noise in hot jets, NASA TM 215274 (2008).

Camussi, R. and Guj, G., Experimental analysis of intermittent coherent structures in the near field of a high Re turbulent jet flow, Physics of Fluids, Vol. 11, No. 2 (1999), pp. 423-431. 
Cuppoletti, D., Gutmark, E., Hafsteinsson, H. and Eriksson, L.-E., The role of nozzle contour on supersonic jet thrust and acoustics, AIAA Journal, Vol. 52, No. 11 (2014), pp. 2594-1614.

Dargaud, J.B., Troyes, J., Lamet, J.-M., Tessé, L., Vuillot, F., and Bailly, C., A numerical study of solid rocket motor ignition overpressure wave including infrared radiation, Journal of Propulsion and Power, Vol. 30, No. 1 (2013), pp. 164-174.

De Cacqueray, N. and Bogey, C., Noise of an overexpanded Mach 3.3 jet: non-linear propagation effects and correlations with flow, International Journal of Aeroacoustics, Vol. 13, No. 7-8 (2014), pp. 607-632.

De Cacqueray, N., Bogey, C. and Bailly, C., Investigation of a high-Mach-number overexpanded jet using large-eddy simulation, AIAA Journal, Vol. 49, No. 10 (2011), pp. 2171-2182.

Edgington-Mitchell, D., Oberleithner, K., Honnerey, D.R. and Soria, J., Coherent structure and sound production in the helical mode of a screeching axisymmetric jet, Journal of Fluid Mechanics, Vol. 748 (2014), pp. 822-847.

Fujii, K., Nonomura, T. and Tsutsumi, S., Toward accurate simulation and analysis of strong acoustic wave phenomena - A review from the experience of our study on rocket problems, International Journal for Numerical Methods in Fluids, Vol. 64 (2010), pp. 1412-1432.

Gao, J.H. and Li, X.D., A multi-mode screech frequency prediction formula for circular supersonic jets, Journal of the Acoustical Society of America, Vol. 127, No. 3 (2010), pp. 1251-1257.

Gee, K.L., Sparrow, V.W., James, M.M., Downing, J.M., Hobbs, C.M., Gabrielson and T.B.Atchley, A.A., The role of nonlinear effects in the propagation of noise from high-power jet aircraft, Journal of the Acoustical Society of America, Vol. 123, No. 6 (2008), pp. 4082-4093.

Gojon, R., Bogey, C. and Marsden, O., Large-eddy simulation of underexpanded round jets impinging on a flat plate 4 to 9 radii downstream from the nozzle, 21st AIAA/CEAS Aeroacoustics Conference, AIAA Paper 2015-2210 (2015).

Harper-Bourne, M. and Fisher, M.J., 1973, The noise from shock waves in supersonic jets, AGARD CP No. 131 (1973), 11.1-11.13.

Harper-Bourne, M., On modelling the near-field noise of the high-speed jet exhausts of combat aircraft, AIAA Paper 2002-2424 (2002).

Heeb, N., Gutmark, E., Liu, J. and Kailasanath, K., Fluidically enhanced chevrons for supersonic jet noise reduction, AIAA Journal, Vol. 52, No. 4 (2014), 799-809.

Henderson, B., Bridges, J. and Wernet, M., 2005, An experimental study of the oscillatory flow structure of tone-producing supersonic impinging jets, Journal of Fluid Mechanics, Vol. 542 (2005), pp. 115-137.

Honda, H., Nonomura, T., Fujii, K., and Yamamoto, M., Effects of Plate Angles on Acoustic Waves from a Supersonic Jet Impinging on an Inclined Flat Plate, 41th AIAA Fluid Dynamics conference and Exhibit, AIAA-2011-3260 (2011). (revised and submitted to AIAA Journal in 2015).

Juvé, D., Sunyach, M. and Comte-Bellot, G., Intermittency of the noise emission in subsonic cold jets, Journal of Sound and Vibration, Vol. 71, No. 3 (1980), pp. 319-332.

Kumar, R., Lazic, S. and Alvi, F.S., Control of high-temperature supersonic impinging jets using microjets, AIAA Journal, Vol. 47, No. 12 (2009), pp. 2800-2811.

Liu, J., Kailasanath, K., Ramamurti, R., Munday, D., Gutmark, E. and Lohner, R., Large-eddy simulations of a supersonic jet and its near-field acoustic properties, AIAA Journal, Vol. 47, No. 8 (2009), pp. 1849-1864.

Liu, J., Kailasanath, K., Boris, J. B., Heeb, N., Munday, D. and Gutmark, E., Effect of the initial turbulence level on an underexpanded supersonic jet, AIAA Journal, Vol. 51, No. 3 (2013), pp. 741-744.

Mora, P., Heeb, N., Kastner, J., Gutmark, E. and Kailasanath, K., Impact of heat on the pressure skewness and kurtosis in supersonic jets, AIAA Journal, Vol. 52, No. 4 (2014), pp. 777-787.

Morris, P.J., Jet noise prediction: past, present and future, Canadian Acoustics, Vol. 35, No. 3 (2007), pp. 16-22.

Morris, P.J., The instability of high speed jets, Int. Journal of Aeroacoustics, Vol. 9, No. 1 \& 2 (2010), pp. 1-50.

Morris, P.J. and Miller, A.E., Prediction of broadband shock-associated noise using Reynolds-averaged Navier-Stokes computational fluid dynamics, AIAA Journal, Vol. 48, No. 12 (2010), pp. 2931-2944.

Munday, D., Gutmark, E., Liu, J. and Kailasanath, K., Flow structure and acoustics of supersonic jets from conical convergent-divergent nozzles, Physics of Fluids, Vol. 23, No. 116102 (2011), pp. 1-13.

Nagata, Y., Nonomura, T., Fujii, K., and Yamamoto, M., Analysis of acoustic-fields generated by a supersonic jet impinging on flat and curved inclined plates, International Journal of Aerospace and Lightweight Structures, Vol. 3 , No. 3 (2013) pp. 357-371.

Nichols, J.W., Lele, S.K., Ham, F.E., Martens, S. and Spyropoulos, J.T., Crackle noise in heated supersonic jets, Journal 
of Engineering for Gas Turbines and Power, Vol. 135, No. 051202 (2013), pp. 1-7.

Nonomura, T. and Fujii, K., Overexpansion effects on characteristics of Mach waves from a supersonic cold jet, AIAA Journal, Vol. 49, No. 10 (2011), pp. 2282-2294.

Nonomura, T., Goto, Y. and Fujii, K., Aeroacoustic waves generated from a supersonic jet impinging on an inclined flat plate, Aeroacoustics, Vol. 10, No. 4 (2011), pp. 401-426.

Norum, T.D., Screech suppression in supersonic jets, AIAA Journal, Vol. 21, No. 2 (1983), pp. 235-240.

Oertel Sen., H., Seiler, F. and Srulijes, J., Visualization of Mach waves produced by supersonic jet and theoretical explanations, Journal of Visualization, Vol. 16, No. 4 (2013), pp. 303-312.

Panda, J., An experimental investigation of screech noise generation, Journal of Fluid Mechanics, Vol. 378 (1999), pp. 7196.

Panda, J., Seasholtz, R.G. and Elam, K.A., Investigation of noise sources in high-speed jets via correlation measurements, Journal of Fluid Mechanics, Vol. 537 (2005), pp. 349-385.

Papamoschou, D., Morris, P.J. and McLaughlin, D.K., Beamformed flow-acoustic correlations in a supersonic jet, AIAA Journal, Vol. 48, No. 10 (2010), pp. 2445-2453.

Ponton, M.K. and Seiner, J.M., The effects of nozzle exit lip thickness on plume resonance, Journal of Sound and Vibration, Vol. 154, No 3 (1992), pp. 531-549.

Powell, A., On the mechanism of choked jet noise, Proc. Phys. Soc. London, Vol. B66 (1953), pp. 1039-1057.

Powell, A., Umeda, Y. and Ishii, R., 1992, Observations of the oscillation modes of choked circular jets, Journal of the Acoustical Society of America, Vol. 92, No. 5 (1992), pp. 2823-2836.

Powell, A., On Prandtl's formulas for supersonic jet cell length, Int. Journal of Aeroacoustics, Vol. 9, Nos. 1 \& 2 (2010), pp. 207-236.

Raman, G., Cessation of screech in underexpanded jets, Journal of Fluid Mechanics, Vol. 336 (1997), pp. 69-90.

Raman, G., Supersonic jet screech: half-century from Powell to the present, Journal of Sound and Vibration, Vol. 225, No. 3 (1999), pp. 543-571.

Ryu, J., Lele, S.K. and Viswanathan, K., Study of supersonic wave components in high-speed turbulent jets using an LES database, Journal of Sound and Vibration, Vol. 333 (2014), pp. 6900-6923.

Seiner, J.M., Advances in high speed jet aeroacoustics, AIAA Paper 84-2275 (1984).

Seiner, J.M. and Yu, J.C., 1984, Acoustic near field properties associated with broadband shock noise, AIAA Journal, Vol. 22 (1984), pp. 1207-1215.

Shen, H. and Tam, C.K.W., Three-dimensional numerical simulation of the jet screech phenomenon, AIAA Journal, Vol. 40, No. 1 (2002), pp. 33-41.

Sinha, A., Kim, K., Kim, J.-H., Serrani, A. and Samimy, M., Extremizing feedback control of a high-speed and highReynolds number jet, AIAA Journal, Vol. 48, No. 2 (2010), pp. 387-399.

Sinha, A., Rodıg̀uez, D., Brès, G. and Colonius, T., Wavepacket models for supersonic jet noise, Journal of Fluid Mechanics, Vol. 742 (2014), pp. 71-95.

Suzuki, T. and Lele, S., Shock leakage through an unsteady vortex-laden mixing layer: application to screech jet, Journal of Fluid Mechanics, Vol. 490 (2003), pp. 139-167.

Tam, C.K.W. and Burton, D.E., Sound generated by instability waves of supersonic flows. Part 2: axisymmetric jets, J. Fluid Mech., Vol. 138 (1984), pp. 273-295.

Tam, C.K.W. and Hu, F.Q., On the three families of instability waves of high-speed jets, Journal of Fluid Mechanics, Vol. 201 (1989), pp. 447-483.

Tam, C.K.W. and Ahuja, K.K., Theoretical model of discrete tone generation by impinging jets, Journal of Fluid Mechanics, Vol. 214 (1990), pp. 67-87.

Tam, C.K.W., Supersonic jet noise, Annual Review of Fluid Mechanics, Vol. 27 (1995), pp. 17-43.

Tam, C.K.W., Golebiowski, M. and Seiner, J.M., On the two component of turbulent mixing noise from supersonic jets. 18th AIAA/CEAS Aeroacoustics Conference, AIAA Paper 96-1716 (1996).

Tam, C.K.W., Viswanathan, K., Ahuja, K.K. and Panda, J., The sources of jet noise: experimental evidence, Journal of Fluid Mechanics, Vol. 615 (2008), pp. 253-292.

Tam, C.K.W., Pastouchenko, N.N. and Viswanathan, K., Computation of shock cell structure of dual-stream jets for noise prediction, AIAA Journal, Vol. 46, No. 11 (2008), pp. 2857-2867.

Tam, C.K.W., Pastouchenko, N.N. and Viswanathan, K., Broadband shock-cell noise from dual stream jets, Journal of Sound and Vibration, Vol. 324 (2009), pp. 861-891. 
Tam, C.K.W., 2009, Mach wave radiation from high-speed jets, AIAA Journal, Vol. 47, No. 10 (2009), pp. $2440-2448$.

Tam, C.K.W., Parrish, S.A. and Viswanathan, K., Harmonics of jet Screech tones, AIAA Journal, Vol. 52, No. 11 (2014), pp. 2471-2479.

Tanna, H.K., An experimental study of jet noise, Part I: turbulent mixing noise, Journal of Sound and Vibration, Vol. 50, No. 3 (1977a), pp. 405-428.

Tanna, H.K., An experimental study of jet noise, Part II: shock associated noise, Journal of Sound and Vibration, Vol. 50, No. 3 (1977b), pp. 429-444.

Tatsukawa, T., Nonomura, T., Oyama, A. and Fujii, K., Nozzle-to-ground distance effect on nondominated solutions of multiobjective aeroacoustic flame deflector design problem, 21st AIAA/CEAS Aeroacoustics Conference, AIAA Paper 2015-2683 (2015).

Tsutsumi, S. Ishii, T. Ui, K. Tokudome, S. and Wada. K., Study on acoustic prediction and reduction of Epsilon launch vehicle at liftoff, Journal of Spacecraft and Rockets, Vol. 52, No. 2 (2015), pp. 350-361.

Viswanathan, K., Aeroacoustics of hot jets, Journal of Fluid Mechanics, Vol. 516 (2014), pp. 39-82.

Viswanathan, K., Scaling laws and a method for identifying component of jet noise, AIAA Journal, Vol. 44, No. 10 (2006), pp. 2274-2285.

Viswanathan, K., Alkislar, M.B. and Czech, M.J., Characteristics of the shock noise component of jet noise, AIAA Journal, Vol. 48, No. 1 (2010), pp. 25-46. 